\title{
Observation of a critical pressure gradient for the stabilization of interchange modes in simple magnetized toroidal plasmas
}

\author{
L. Federspiel, B. Labit, P. Ricci, A. Fasoli, I. Furno, and C. Theiler \\ Centre de Recherches en Physique des Plasmas (CRPP), Ecole Polytechnique Fédérale de Lausanne \\ (EPFL), Association EURATOM-Confédération Suisse, CH-1015 Lausanne, Switzerland
}

(Received 3 April 2009; accepted 24 July 2009; published online 3 September 2009)

\begin{abstract}
The existence of a critical pressure gradient needed to drive the interchange instability is experimentally demonstrated in the simple magnetized torus TORoidal Plasma EXperiment [A. Fasoli et al., Phys. Plasmas 13, 055902 (2006)]. This gradient is reached during a scan in the neutral gas pressure $p_{n}$. Around a critical value for $p_{n}$, depending on the magnetic configuration and on the injected rf power, a small increase in the neutral gas pressure triggers a transition in the plasma behavior. The pressure profile is locally flattened, stabilizing the interchange mode observed at lower neutral gas densities. The measured value for the critical gradient is close to the linear theory estimate. [doi:10.1063/1.3204704]
\end{abstract}

\section{INTRODUCTION}

In simple magnetized tori (SMT), global interchange modes characterized by $k_{\|} \simeq 0$ (flutelike) can be destabilized by pressure gradients in the unfavorable curvature region, where $\nabla B$ and $\nabla p$ are collinear. Linear theories developed for $Z$ - pinch $^{1,2}$ and magnetic dipole ${ }^{3,4}$ configurations have shown that plasma compressibility can stabilize the interchange mode even in the presence of a finite pressure gradient.

The aim of this paper is to determine whether a critical pressure gradient for interchange instabilities exists in the SMT device TORPEX [TORoidal Plasma EXperiment (Ref. 5)] and to experimentally quantify its value. In TORPEX, a vertical magnetic field $B_{z}$ is superposed to a toroidal field $B_{\varphi}$, creating helical field lines with both ends terminating on the torus vessel. TORPEX is particularly well suited for the quest of a critical pressure gradient. Its flexibility, together with the high spatial and time resolved set of diagnostics, led to the characterization of linear and nonlinear properties for low frequency electrostatic instabilities ${ }^{5-7}$ and of the associated transport. ${ }^{8,9}$ Herein the existence of a threshold gradient driving the interchange instability observed in certain TORPEX plasmas is experimentally demonstrated during a scan in the neutral gas pressure $p_{n}$. Around a critical neutral concentration $p_{n \text {,crit }}$, a small increase in the latter causes a profile broadening and a reduction in the fluctuation amplitude. The pressure profile is flattened locally, stabilizing the interchange mode observed at lower $p_{n}$. The value of the critical pressure gradient measured experimentally is in agreement with the theoretical prediction.

The outline of the paper is as follows. In Sec. II, the existence of a critical gradient for the interchange instability is derived from linear theory. The experimental setup and the typical TORPEX plasmas are described in Sec. III, along with the identification of the instability. The experimental evidence for the existence of a critical gradient obtained from the neutral gas pressure scan is presented in Sec. IV. Finally, a discussion of the results implications for possible further developments concludes the paper (Sec. V).

\section{THEORETICAL BACKGROUND}

For the SMT configuration, we consider a linearized system for the plasma density $n$, the electron temperature $T_{e}$, and the plasma potential $\phi$, based on the two-dimensional (2D) Braginskii drift-reduced equations in the limit $\beta=2 \mu_{0} \bar{n} \bar{T}_{e} / B^{2} \ll 1, T_{i} \ll T_{e}$, and $k_{\|}=0 .{ }^{2,10}$ Drift wave terms are neglected since our study concentrates on interchange modes. We denote each quantity as the sum of a stationary and a fluctuating part: $f=\bar{f}+\tilde{f}$. The equations are written in a coordinate system parallel to the magnetic field lines, where $x$ is the radial direction and $y$ is the direction perpendicular to $x$ and the magnetic field line. Assuming no stationary flows $(\bar{\phi}=0)$ and including an ion-neutral collision term, the linearized equations for the fluctuating quantities $\widetilde{n}, \widetilde{\phi}$, and $\widetilde{T}_{e}$ can be deduced from the model in Ref. 11. At the radial position $R$, the linear system is given by

$$
\begin{aligned}
\frac{\partial \widetilde{n}}{\partial t}= & -\frac{1}{B} \frac{\partial \widetilde{\phi}}{\partial y} \frac{\partial \bar{n}}{\partial x}+\frac{2}{e R B}\left(\bar{n} \frac{\partial \widetilde{T}_{e}}{\partial y}+\bar{T}_{e} \frac{\partial \widetilde{n}}{\partial y}-e \bar{n} \frac{\partial \widetilde{\phi}}{\partial y}\right) \\
& +\frac{\omega_{\sigma}}{\bar{T}_{e}}\left[\overline{T_{e}} \widetilde{n}+\left(\Lambda+\frac{1}{2}\right) \widetilde{T}_{e}-e \widetilde{\phi}\right], \\
\frac{\partial \nabla^{2} \widetilde{\phi}}{\partial t}= & -\nu_{i n} \nabla^{2} \widetilde{\phi}+\frac{2 B}{m_{i} R}\left(\frac{\overline{T_{e}}}{\bar{n}} \frac{\partial \widetilde{n}}{\partial y}+\frac{\partial \widetilde{T}_{e}}{\partial y}\right) \\
& -\frac{\omega_{\sigma}}{\overline{T_{e}}} \frac{\Omega_{i}^{2}}{e}\left(\Lambda \widetilde{T}_{e}-e \widetilde{\phi}\right), \\
\frac{\partial \widetilde{T}_{e}}{\partial t}= & -\frac{1}{B} \frac{\partial \widetilde{\phi}}{\partial y} \frac{\partial \overline{T_{e}}}{\partial x}+\frac{4}{3 e R B}\left(\frac{7}{2} \overline{T_{e}} \frac{\partial \widetilde{T}_{e}}{\partial y}+\frac{\overline{T_{e}^{2}}}{\bar{n}} \frac{\partial \widetilde{n}}{\partial y}-e \overline{T_{e}} \frac{\partial \widetilde{\phi}}{\partial y}\right) \\
& -\frac{2}{3} \omega_{\sigma}\left[\left(1.71 \Lambda+\frac{3}{2}\right) \widetilde{T}_{e}-1.71 e \widetilde{\phi}\right] .
\end{aligned}
$$

In Eq. (2) the ion-neutral collision frequency $\nu_{\text {in }} \simeq n_{n} \sigma_{\text {ion }} v_{\text {thi }}=p_{n} / T_{\mathrm{amb}} \sigma^{m t} v_{\text {thi }}$ is introduced, where $p_{n}$ is the neutral background pressure, $T_{\text {amb }}$ is the neutral gas tempera- 
ture, and $\sigma^{m t}$ is the momentum transfer cross section. ${ }^{12}$ The electron-neutral collision term can be neglected compared to $\nu_{i n}$ because of the mass ratio $m_{e} / m_{i}$. In the linearized system the terms related to the interaction of the plasma with the vessel are those proportional to $\omega_{\sigma}=(\sigma / R) \sqrt{\bar{T}_{e} / m_{i}}$, with $\sigma=\Delta /\left(2 \pi L_{v}\right)=\left(B_{z} / B_{\varphi}\right)\left(R / L_{v}\right), \Delta=2 \pi R\left(B_{z} / B_{\varphi}\right)$ and the SMT height $L_{v}$. The term $\Lambda$ defines the Coulomb logarithm $\Lambda=\log \sqrt{m_{i} /\left(2 \pi m_{e}\right)}$ and $\Omega_{i}$ corresponds to the ion-cyclotron frequency. Each fluctuating quantity can be written in the form: $\tilde{f}=\tilde{f} \exp \left(\gamma t+i k_{y} y\right)$, where $\gamma=-i \omega$ and the real part $\mathfrak{R}(\gamma)$ is the growth rate. The mode is driven unstable when $\mathfrak{R}(\gamma)>0$. Moreover, we consider a local approximation, i.e., that the perturbations do not depend on $x$.

A dispersion relation for interchange modes, including the ion-neutral collision term $\nu_{i n}$ is derived from the linearized system [Eqs. (1)-(3)]:

$$
b_{00} \nu_{i n}+b_{0}+\left(b_{1}+b_{2} \nu_{i n}\right) \gamma+\left(b_{2}+b_{3} \nu_{i n}\right) \gamma^{2}+b_{3} \gamma^{3}=0,
$$

with $\quad b_{00}=(20 / 3) \omega_{d}^{2} k_{y}^{2} \rho_{s}^{2}, \quad b_{0}=(20 / 3) i \omega_{d}^{2}\left(2 \omega_{d}+\omega_{*}\right)$, $b_{1}=(20 / 3)\left(k_{y}^{2} \rho_{s}^{2}-1\right) \omega_{d}^{2}-2(\eta+1) \omega_{*} \omega_{d}, \quad b_{2}=(20 / 3) i \omega_{d} k_{y}^{2} \rho_{s}^{2}$, $b_{3}=-k_{y}^{2} \rho_{s}^{2}$, and where $\omega_{d}=k_{y} \rho_{s} c_{s} / R$ is the curvature frequency, $\omega_{*}=k_{y} \rho_{s} c_{s} / L_{n}$ is the diamagnetic frequency, $\eta=L_{n} / L_{T}$, with $L_{n}=\bar{n} /(d \bar{n} / d x)$ and $L_{T}=\overline{T_{e}} /\left(d \overline{T_{e}} / d x\right)$. The $\omega_{\sigma}$ term can be neglected in Eq. (4), since for the experimental scenarios considered here $\omega_{\sigma} \ll \omega_{d}, \omega_{*}$ and $\nu_{i n}$. A similar dispersion relation, but neglecting the ion-neutral collision term, has been derived in Ref. 2 .

Assuming $k_{y} \rho_{s} \ll 1$ (scaling $\gamma \sim c_{s} / R$ ), Eq. (4) reduces to

$$
\left[k_{y}^{2} \rho_{s}^{2} \gamma^{2}+\nu_{i n} k_{y}^{2} \rho_{s}^{2} \gamma+\left(\frac{20}{3} \omega_{d}^{2}+2(\eta+1) \omega_{*} \omega_{d}\right)\right] \gamma=0 .
$$

The trivial solution $\gamma=0$ has no interest. A root of Eq. (5) with positive real part only exists if the third term in the square brackets is negative. Thus, the condition for instability reads

$$
\frac{20}{3} \omega_{d}^{2}+2(\eta+1) \omega_{*} \omega_{d}<0
$$

This condition does not depend on $\nu_{i n}$ : the ion-neutral collisions do not affect the value of the critical normalized pressure gradient $L_{p}=\bar{p}_{e} /\left(d \bar{p}_{e} / d x\right)$ for interchange instabilities. Introducing $L_{p}^{-1}=(\eta+1) L_{n}^{-1}$ in Eq. (6) we get a stability condition. Interchange modes are stable if

$$
L_{p}^{-1}>L_{p, \text { crit }}^{-1}=-\frac{10}{3 R} .
$$

With $R=1 \mathrm{~m}$ for the TORPEX device, we get $L_{p \text {, crit }}^{-1} \cong-3.3 \mathrm{~m}^{-1}$. An interchange mode cannot be driven unstable on the HFS since $L_{p}$ is always positive in that region. On the LFS, interchange modes are driven unstable if $L_{p}^{-1}<L_{p \text {,crit }}^{-1}$ One has to note that the interchange mode frequency in the laboratory frame is essentially given by the $E \times B$ drift velocity $\left(\omega_{I} \cong \omega_{E \times B}=k_{\perp} v_{E \times B}\right)$. Therefore, a change in the $E \times B$ flow profile locally affects the measured mode frequency. However, a constant $E \times B$ flow, not included in Eqs. (1)-(3), does not modify the threshold value we found. Shear flows are not considered in this analysis, even though they could affect the instability threshold. Note also that, contrary to the interchange, for drift modes it can be shown that collisions affect the pressure radial profile and thus the pressure gradient threshold. ${ }^{13,14}$

To address the question of the existence of a critical pressure gradient experimentally, one needs to find a way to efficiently decrease the pressure gradient until interchange modes are stabilized. If a critical gradient is observed, its value can be compared with the prediction of the linear theory.

\section{EXPERIMENTAL SETUP AND STANDARD PLASMAS}

The experiments are performed in the simple magnetized toroidal device TORPEX (Ref. 5) (major radius $R=1 \mathrm{~m}$ and minor radius $a=0.2 \mathrm{~m}$ ). Helical magnetic field lines are obtained by superposing a vertical magnetic field $B_{z} \cong 1.3 \mathrm{mT}$ to a dominant toroidal magnetic field $B_{\varphi} \cong 80 \mathrm{mT}$, which can be slightly varied in order to maintain the plasma at the center of the poloidal section. Plasmas of different gases $\left(\mathrm{H}_{2}, \mathrm{He}, \mathrm{Ne}, \mathrm{Ar}, \mathrm{Kr}, \ldots\right)$ can be produced and sustained by continuous injection of microwaves in the range of electron cyclotron $(\mathrm{EC})$ frequencies $(2.45 \mathrm{GHz})$. As rf power is an efficient tool to control the density profile, ${ }^{15} \mathrm{a}$ low power magnetron $\left(P_{\mathrm{rf}} \leq 1.2 \mathrm{~kW}\right)$ is used in order to produce low density and weakly turbulent plasmas, as needed for these specific experiments. We focus on neon plasmas, for which we expect stronger collisional effects than for lighter ions. The neutral gas pressure can be varied between $2 \times 10^{-5}$ and $2 \times 10^{-3}$ mbar. Measurements are taken with the 2D 86 Langmuir probes (LP) fixed array HEXTIP (with a $3.5 \mathrm{~cm}$ pin separation ${ }^{16}$ ), the eight-tip LP movable array SLP and an eight-tip array fixed near the vessel walls on the LFS. The LP can be operated in the ion saturation current regime $\left(V_{\text {bias }}=-40 \mathrm{~V}\right)$ or in the swept mode needed to extract the time averaged profiles.

The time averaged 2D density profile of a typical plasma, measured with HEXTIP is shown in Fig. 1(a). The neutral pressure is $p_{n}=7.6 \times 10^{-4}$ mbar with $P_{\mathrm{rf}} \cong 120 \mathrm{~W}$, $B_{z} \cong 1.3 \mathrm{mT}$, and $B_{\varphi} \cong 80 \mathrm{mT}$. The density profile is directly derived from measurements of the ion saturation current, assuming a constant electron temperature of $5 \mathrm{eV}$. The plasma is centered around $r=-2 \mathrm{~cm}\left(n_{e, \max } \simeq 3.7 \times 10^{16} \mathrm{~m}^{-3}\right)$ and is elongated in the vertical direction (slablike). A coherent mode with $f \cong 7.5 \mathrm{kHz}$ is detected at $r=3.5 \mathrm{~cm}$ and $z=0 \mathrm{~cm}$ [Fig. 1(c)]. The 2D profile of the rms of $\tilde{n}_{e}$ calculated in the range $\Delta f=[3.4,12] \mathrm{kHz}$ and normalized over the entire spectrum ${ }^{7}$ shows that this mode is observed for all $z$ in the region $0 \leq r \leq 10 \mathrm{~cm}$ on the LFS [Fig. 1(b)].

To identify the electrostatic instability observed in this plasma, the dispersion relation of the wave is evaluated across and along the magnetic field from the wavenumberfrequency power spectrum $P\left(k_{z}, \omega\right)$ computed for density fluctuations using a two point correlation technique. ${ }^{17,18}$ In TORPEX, this technique has been used to identify instabilities in hydrogen plasmas. ${ }^{7}$ We apply it here to characterize neon plasmas. Indeed, changing the mass ratio $m_{e} / m_{i}$ and the plasma resistivity might affect the properties of drift waves. It is thus essential to verify that the investigated plasmas 


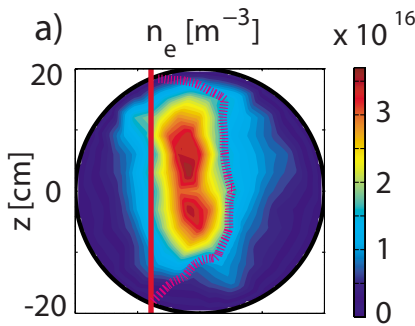

b) $\operatorname{rms}\left(\tilde{\mathrm{n}}_{\mathrm{e}, \Delta f}\right) / \mathrm{rms}\left(\tilde{\mathrm{n}}_{\mathrm{e}, \mathrm{tot}}\right)$
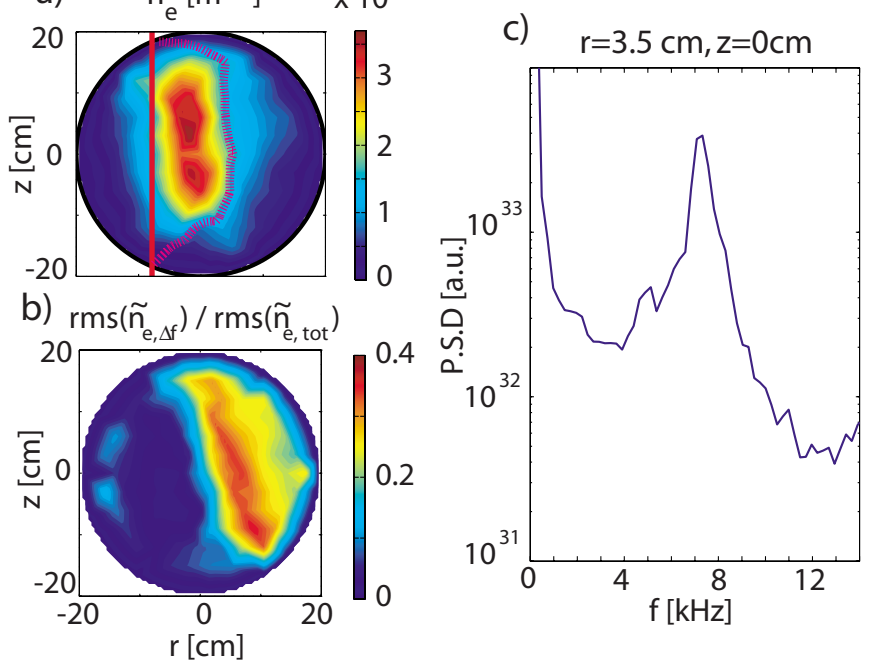

FIG. 1. (Color online) 2D time averaged profiles of (a) the electron density $n_{e}$, (b) the rms of the electron density $\widetilde{n}_{e}$ in the range $\Delta f=[3.4,12] \mathrm{kHz}$ normalized over the entire spectrum, and (c) power spectral density for $I_{\text {sat }}$ measured at $r=3.5 \mathrm{~cm}$.

are still in an interchange dominated regime. To apply this method, the distance between the two LPs measuring $\tilde{I}_{\text {sat }}$ has to be much smaller than the correlation length. The conditional wavenumber-frequency spectrum $p\left(k_{z} \mid \omega\right)$ $=P\left(k_{z}, \omega\right) / \Sigma_{k_{z}} P\left(k_{z}, \omega\right)$ is evaluated at $r=7 \mathrm{~cm}$ with two probes separated in the vertical direction by a distance $\Delta z=1.34 \mathrm{~cm}$ [Fig. 2(a)]. Figure 2(b) shows the mean value $\bar{k}_{z}(\omega)$ and the variance $\bar{\sigma}_{k_{z}}(\omega)$ of the spectral density in the wavenumber space. The vertical wavenumber $k_{z}$ associated with the frequency of the mode $7.5 \pm 0.6 \mathrm{kHz}$ is about $k_{z}=(50.4 \pm 7.4) \mathrm{m}^{-1}$. This corresponds to a wavelength $\lambda_{z} \simeq 12.5 \mathrm{~cm}$, which is approximately equal to the vertical displacement of the magnetic field lines after one toroidal turn at this radial location $(\Delta \simeq 11.5 \mathrm{~cm}$ for $R=1.07 \mathrm{~m})$. The mode phase velocity $v_{p h}=\Sigma_{k_{z}, \omega}\left(\omega / k_{z}\right) P\left(k_{z}, \omega\right) / \Sigma_{k_{z}, \omega} P\left(k_{z}, \omega\right)$ is estimated to be $v_{p h}=(920 \pm 73) \mathrm{m} / \mathrm{s}$, which is in good agreement with the $E \times B$ drift velocity (not shown). The estimated vertical wavelength and the phase velocity are indicators that the observed instability is an interchange mode. To complete the mode identification, one needs to estimate the parallel wavenumber. This is done by measuring the phase shift between two toroidally separated $(\Delta \varphi=\pi / 2)$ probes and positioned as precisely as possible on the same magnetic field line. The details of this procedure are given in Refs. 7, 19, and 20. Figures 2(c) and 2(d) summarize the measurements of the parallel wavenumber at $r=7 \mathrm{~cm}$. The high level of the spectral coherence estimated at the mode frequency confirms the quality of our measurements [Fig. 2(c)]. Figure 2(d) shows the linear relation between the measured wavenumber and the corresponding vertical distance separating the tips. For the optimal vertical separation defined as $z_{\mathrm{opt}}=R \Delta \varphi\left(B_{z} / B_{\varphi}\right)$, a linear fit gives $k_{\|}=(0.02 \pm 0.09) \mathrm{m}^{-1}$, which corresponds to a flute mode within experimental errors. We conclude that the coherent fluctuations observed at $7.5 \mathrm{kHz}$ correspond to an inter-

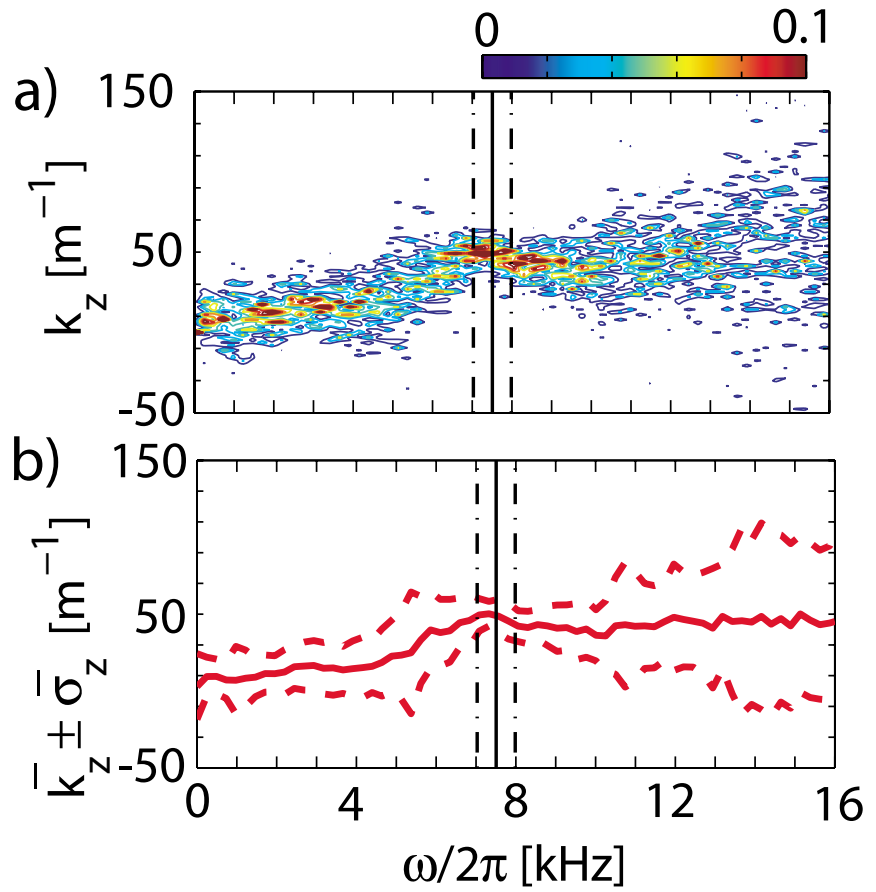

c)
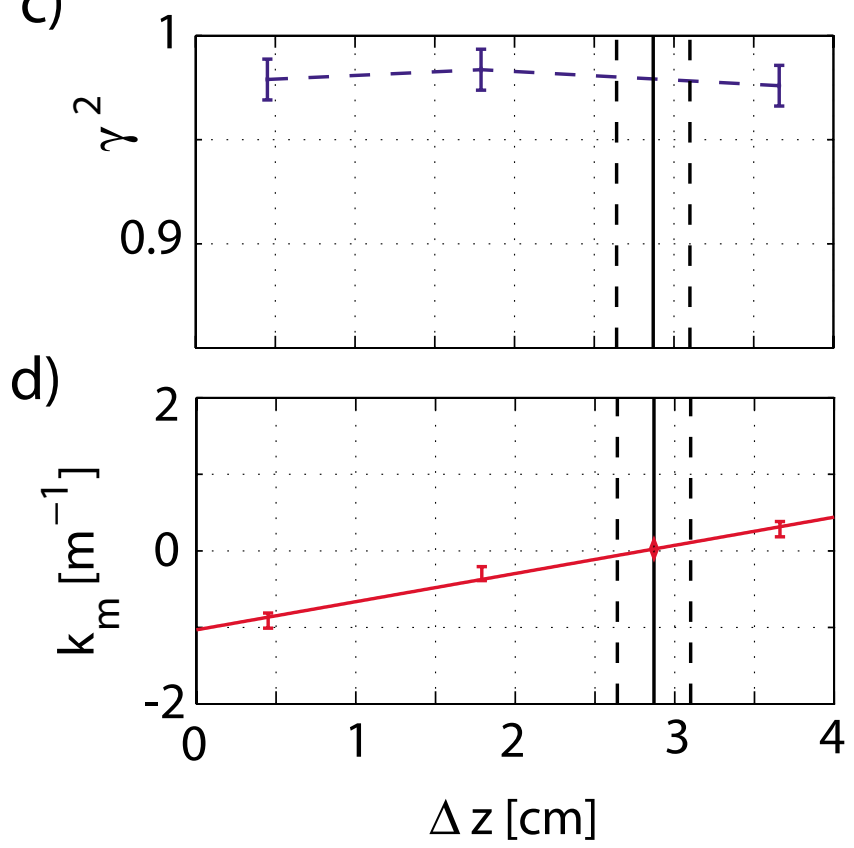

FIG. 2. (Color online) Statistical dispersion relation reconstructed for the mode detected on $I_{\text {sat }}$ measured at $r=7 \mathrm{~cm}$ and $z=0 \mathrm{~cm}$. (a) Conditional wavenumber-frequency spectrum $p\left(k_{z} \mid \omega\right)$. (b) First (thick red solid line) and second (thick red dashed line) moments of the spectral density in the wavenumber space $\bar{k}_{z}(\omega) \pm \bar{\sigma}_{k_{z}}(\omega)$. In (a) and (b) the thin vertical black lines correspond to the mode frequency $\omega_{0} / 2 \pi \pm \Delta \omega_{0} / 2 \pi$. (c) Spectral coherence and (d) wavenumber measured at the frequency of the mode and as a function of the vertical separation between the tips $\left[k_{\|}=k_{m}\left(z_{\text {opt }}\right)\right]$. The error bars associated to $k_{m}$ correspond to the spectral width of the spectrum $P\left(k_{m}\right)$ measured for each couple of tips, whereas the vertical black lines in (c) and (d) define $z_{\text {opt }} \pm \Delta z_{\text {opt }}$.

change mode. The theoretical prediction developed in the previous section can thus be tested in this case.

\section{EXPERIMENTAL EVIDENCE OF A CRITICAL PRESSURE GRADIENT}

Starting from the plasma conditions described in Sec. III, we perform a neutral gas pressure scan between 


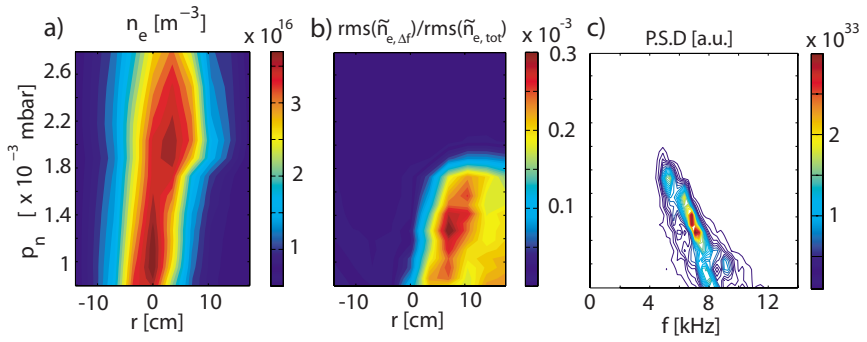

FIG. 3. (Color online) Radial profiles taken at $z=0 \mathrm{~cm}$ of (a) the electron density $n_{e}$, (b) the normalized rms of $\tilde{n}_{e}$ in the range $\Delta f=[3.4,8] \mathrm{kHz}$ for different neutral gas pressures, and (c) frequency spectrum measured at $R-R_{n_{\max }}=7 \mathrm{~cm}$ and $z=0 \mathrm{~cm}$ for different neutral gas pressures.

$p_{n}=0.8 \times 10^{-3} \mathrm{mbar}$ and $p_{n}=2.8 \times 10^{-3} \mathrm{mbar} \quad\left(P_{\mathrm{rf}}\right.$ $\cong 120 \mathrm{~W}$ ). Figure 3 (a) shows the density radial profiles taken at the position $z=0 \mathrm{~cm}$ (from HEXTIP) for various neutral pressures values. It is clearly visible that for neutral pressures lower than $p_{n \text {,crit }}=1.8 \times 10^{-3}$ mbar the density profile depends weakly on $p_{n}$, except that it is shifted to the LFS. As soon as this neutral pressure value is exceeded, an increase in the plasma density is observed, together with a broadening of the profile. For larger neutral gas concentrations, the profile shape remains constant although the total density decreases, which might be due to a less efficient ionization process or to an increase in the plasma losses. This change occurs rather abruptly as the pressure is incremented by $5 \%$ around $p_{n}=1.8 \times 10^{-3} \mathrm{mbar}$, as is evident in the rms of $\tilde{n}_{e}$ calculated in the range $\Delta f=[3.4,8] \mathrm{kHz}$ and normalized over the entire spectrum [Fig. 3(b)]. Below $p_{n \text {,crit, the }}$ frequency of the strong interchange mode decreases with the neutral pressure from 7.5 to $4.8 \mathrm{kHz}$ [Fig. 3(c)]. This is due to a local reduction in the $E \times B$ drift velocity. At the neutral pressure $p_{n \text {,crit }}$ associated with the increase in the electron density $n_{e}$, the interchange mode abruptly disappears.

This transition is relatively robust, since it can occur at a different critical neutral gas pressure for different rf power levels and/or vertical magnetic field values and it has also been observed in argon plasmas. The following trend is observed: the critical neutral gas pressure increases with $B_{z}$ and diminishes when the rf power is incremented as shown in Fig. 4. For similar $B_{z}$ and $P_{\mathrm{rf}}$, the transition occurs at lower

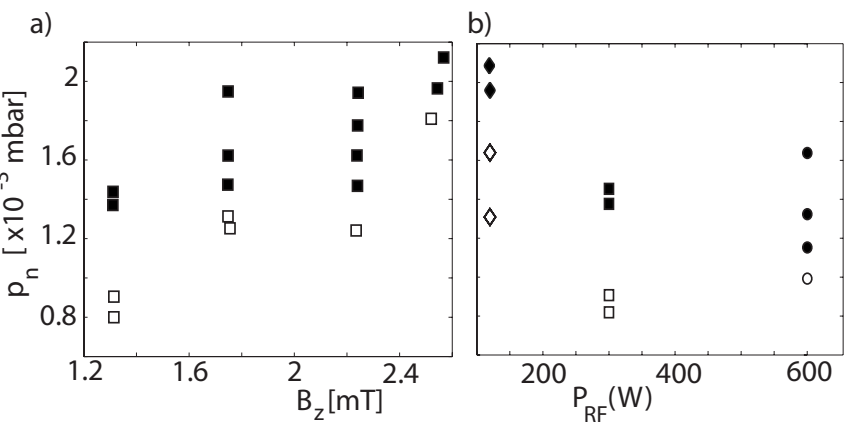

FIG. 4. Neon plasmas below (open symbols) and above (filled symbols) the transition for different neutral gas pressures as a function of (a) the vertical magnetic field $\left(P_{\mathrm{rf}}=300 \mathrm{~W}\right)$ and $(\mathrm{b})$ the rf power $\left(B_{z}=1.3 \mathrm{mT}\right)$. For the $P_{\mathrm{rf}}$ scan, the toroidal magnetic field was varied in order to keep the plasma at the center of the poloidal section: $B_{\varphi}=\{72(\bigcirc) ; 76(\square) ; 80(\diamond)\} \mathrm{mT}$.
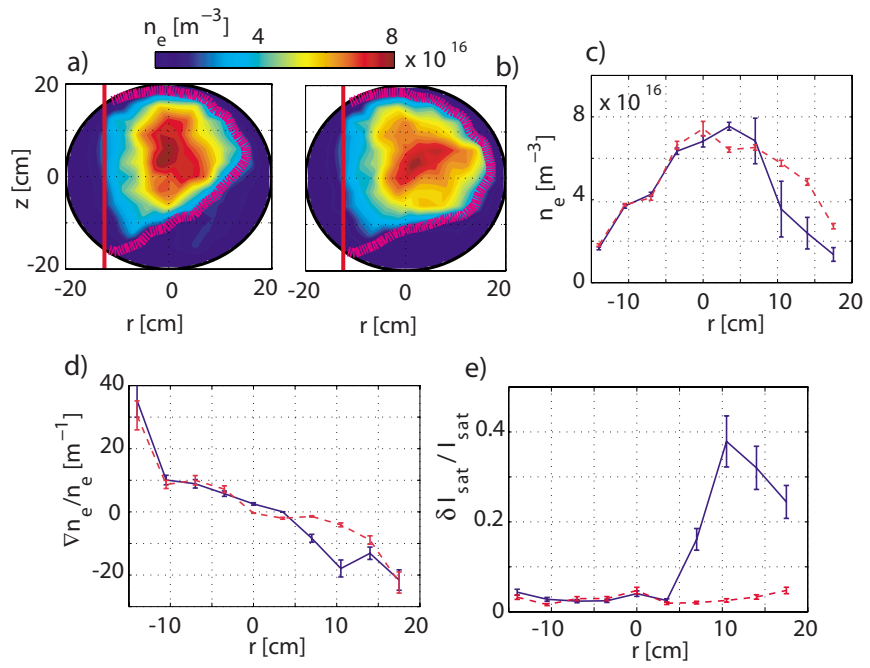

FIG. 5. (Color online) 2D time averaged profile of the density measured with HEXTIP for (a) $p_{n}=1.2 \times 10^{-3} \mathrm{mbar}$ and (b) $p_{n}=1.4 \times 10^{-3} \mathrm{mbar}$. The thick red and the pink dotted lines correspond to the EC (at $R-R_{o}$ $=-12.5 \mathrm{~cm})$ and $\mathrm{UH}$ resonance layers, respectively. Radial profiles measured at $z=0 \mathrm{~cm}$ of (c) the density, (d) the normalized density gradient, and (e) the ratio $\delta I_{\text {sat }} / I_{\text {sat }}$ for the low pressure (solid, blue) and for the high pressure (dashed, red) cases. The error bars correspond to the standard deviation of the time varying quantities.

neutral gas pressures in argon compared to the neon case.

To better characterize the transition, two plasma discharges produced with $P_{\mathrm{rf}} \cong 300 \mathrm{~W}$ have been investigated in detail. In this case, we observe $p_{n, \text { crit }}=1.3 \times 10^{-3}$ mbar. The first plasma is produced using a neutral pressure just below the critical value $\left(p_{n}=1.2 \times 10^{-3} \mathrm{mbar}\right)$, the other with a pressure just above $\left(p_{n}=1.4 \times 10^{-3} \mathrm{mbar}\right)$. The corresponding time averaged density profiles shown in Figs. 5(a) and 5(b) confirm the abrupt increase of the plasma density on the LFS. As a consequence of such increment, the upper hybrid (UH) resonance layer is shifted by a few centimeters toward the LFS [represented by the pink dotted line in Figs. 5(a) and 5(b)] while the level of absorbed microwave power does not change significantly. We remind that in the TORPEX device most of the plasma is generated at the UH resonance layer, where the power absorption is much higher than at the EC layer, once the ionization is initiated. ${ }^{15}$ We cannot exclude that the broadening of the density profile is due to a change in the ionization source, which may be subject for future work. The broadening of the density profile is also visible on the cut at $z=0 \mathrm{~cm}$ shown in Fig. 5(c). It is worth noting

that the profile does not change significantly on the HFS. The profile broadening is associated with the reduction in the normalized density gradient $L_{n}^{-1}$ in the region $6 \leq r \leq 13 \mathrm{~cm}$ [Fig. 5(d)]. As a consequence, the level of fluctuations is reduced by up to a factor of 10 , as shown in Fig. 5(e).

Time averaged 2D profiles of the density $n_{e}$, the electron temperature $T_{e}$, and the plasma potential $V_{p}$ have been reconstructed using the eight-tip movable LP array on a shot-toshot basis. Typical values are $n_{e, \max } \cong 9 \times 10^{16} \mathrm{~m}^{-3}$, $T_{e, \text { max }} \cong 6 \mathrm{eV}$, and $V_{p l, \text { max }} \cong 18 \mathrm{~V}$ [Figs. 6(a) and 6(b)]. Figure 6(c) presents the radial profile of the plasma pressure 

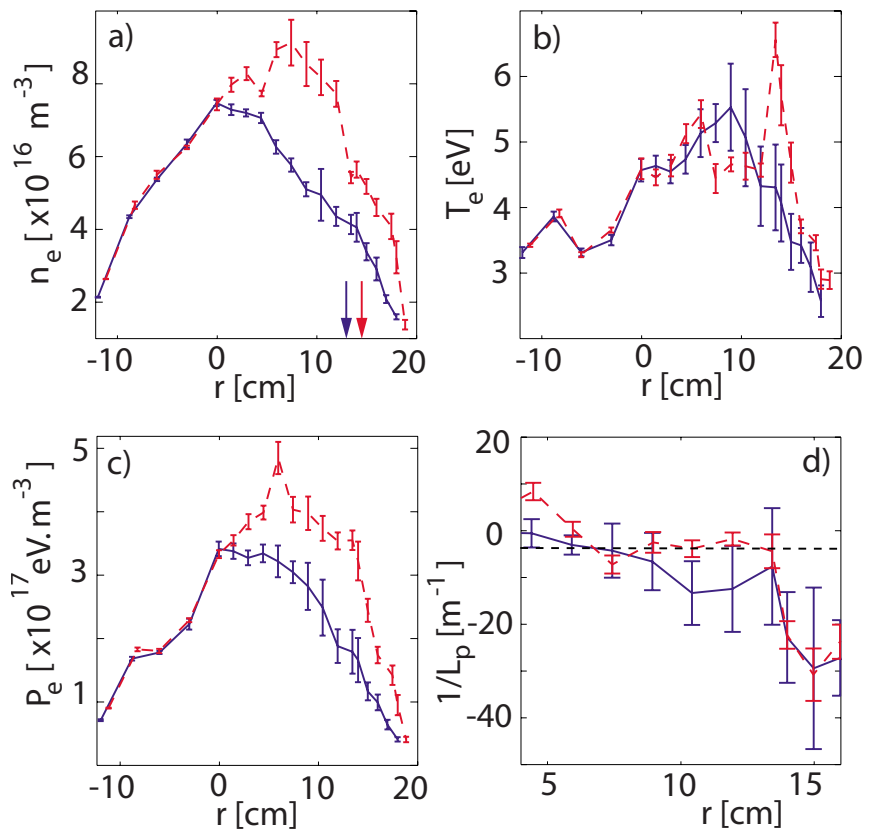

FIG. 6. (Color online) Radial profile of (a) the plasma density, (b) the electron temperature, (c) the plasma pressure $p_{e}$, and (d) the normalized pressure gradient $L_{p}^{-1}=\nabla p_{e} / p_{e}$ for $p_{n}=1.2 \times 10^{-3} \mathrm{mbar}$ (solid, blue) and $p_{n}=1.4 \times 10^{-3} \mathrm{mbar}$ (dashed, red). In (d) the horizontal black dashed line represents the critical value associated with the mode stabilization found in Sec. II [see Eq. (7)]. The error bars are given by the quality of the $I-V$ characteristics fit. In subplot (a) the arrows represent the UH resonance location for both cases $(r=13 \mathrm{~cm}$ for the lower pressure and $r=14.6 \mathrm{~cm}$ for the higher pressure).

$p_{e}=n_{e} T_{e}$ taken at $z=0 \mathrm{~cm}$. Similar to the density profile, a strong flattening in the region $5 \leq r \leq 15 \mathrm{~cm}$ is observed for the higher pressure, while the profile does not change on the HFS. We can therefore exclude any artifact due to the assumption of constant temperature for the $I_{\text {sat }}$ measurements. In quantitative terms, the normalized pressure gradient $\left|L_{p}^{-1}\right|$ is reduced by a factor of four at $r=11 \mathrm{~cm}$ [from $\left|L_{p}^{-1}\right| \cong 13 \mathrm{~m}^{-1}$ to $\left|L_{p}^{-1}\right| \cong 3 \mathrm{~m}^{-1}$, see Fig. 6(d)]. Note that the latter value is close to the $\left|L_{p \text {,crit }}^{-1}\right|$ found in Eq. (7). Together with the time averaged profiles, the properties of the fluctuations are strongly affected by a small increase in the neutral concentration. In particular, the coherent features of the fluctuations spectrum display an abrupt change. The rms of $\tilde{n}_{e}$ in the range $\Delta f=[3.4,8] \mathrm{kHz}$ normalized over the entire spectrum has been estimated for both neutral gas pressures and over the entire plasma cross section (Fig. 7). As expected, for the higher pressure, a strong reduction in the fluctuations amplitude is observed. Moreover, the spatial extent of the fluctuations, which are concentrated only at the very edge in the LFS region, is reduced. The power spectral densities estimated at $r=10.5 \mathrm{~cm}$ and $r=14 \mathrm{~cm}(z=0 \mathrm{~cm})$ reveal the interchange mode at $5.8 \mathrm{kHz}$ for the lower pressure. When the critical neutral gas pressure is exceeded, the mode at $r=10.5 \mathrm{~cm}$ is not detected anymore. A peak is still visible at $r=14 \mathrm{~cm}$ with a $6.4 \pm 0.3 \mathrm{kHz}$ frequency, but with an amplitude reduced by two orders of magnitude (Fig. 7).

It is important to note that the mode detected after the transition maintains its interchange character, as verified on the basis of the dispersion relation for $k_{z}(\omega)$. Its phase veloc-
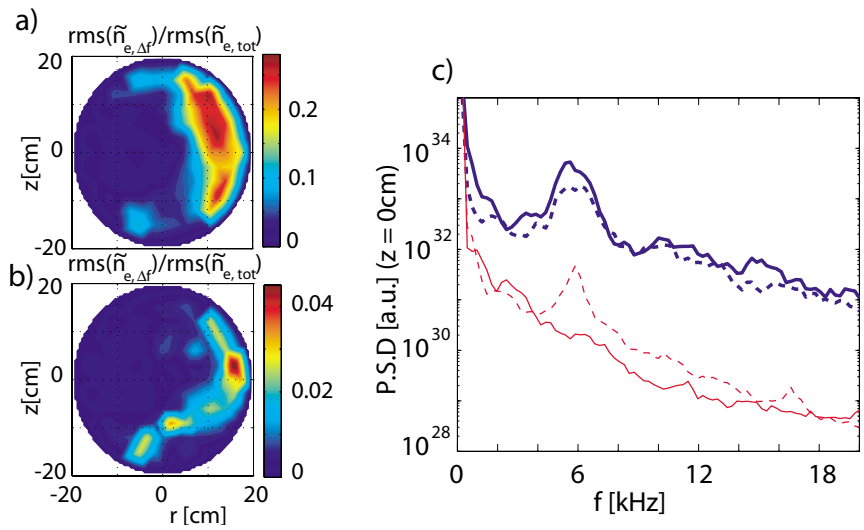

FIG. 7. (Color online) 2D profile of the rms $\tilde{n}_{e}$ in the range $\Delta f=[3.4,8] \mathrm{kHz}$ normalized over the entire spectrum for (a) $p_{n}=1.2$ $\times 10^{-3}$ mbar and (b) $p_{n}=1.4 \times 10^{-3}$ mbar. (c) Power spectral densities for $I_{\text {sat }}$ measured at $r=10.5 \mathrm{~cm}$ (solid) and $r=14 \mathrm{~cm}$ (dashed) for the lower pressure (thick blue) and the higher pressure (thin red).

ity is qualitatively in good agreement with the $E \times B$ drift velocity.

\section{CONCLUDING REMARKS}

In this paper, we investigated the interchange mode linear stability in neon plasmas. The instability grows on top of profiles resulting from a nontrivial combination of several ingredients: a broadened source profile, an $E \times B$ drift velocity with both radial and vertical components, parallel losses with a more complicated pattern than in the slablike configuration, diffusive radial transport due to the high level of neutrals, and finally interchange dynamics. In this work, we did not address the role of the interchange mode as a transport mechanism to establish the plasma profiles.

We have shown that interchange modes can be stabilized in simple magnetized TORPEX plasmas. If a critical neutral gas pressure $p_{n \text {,crit }}$ is exceeded, it is possible to locally $(10 \leq r \leq 14 \mathrm{~cm})$ reduce the pressure gradient below a threshold value. Around the critical $p_{n \text {,crit }}$, depending on the rf power and on the applied magnetic fields $B_{z}$ and $B_{\varphi}$, an increase by only $\sim 5 \%$ of the neutral gas pressure is sufficient to trigger these strong changes in the pressure profile. The observations indicate a bifurcation rather than a smooth transition, suggestive of a critical phenomenon. The experimental critical normalized pressure gradient $L_{p \text {,crit }}^{-1}$ needed to stabilize the interchange instability is in good agreement with the linear threshold estimated in this paper.

The exact role of neutral gas pressure in the mechanism leading to this transition is not easily identifiable and will be the subject of future work. In addition, we note that a recent study on a magnetic dipole device has shown a similar effect of the neutral gas pressure on the low-frequency instabilities. $^{21}$

After the transition, the pressure gradient closer to the edge is almost unchanged and still sufficient to drive the interchange mode. Nevertheless, in this region $(r>13 \mathrm{~cm})$, the level of fluctuations is reduced by two orders of magnitude. Among several mechanisms that could cause this reduction, the $E \times B$ velocity shear might play a significant 
role, as predicted by nonlinear theory and simulations. ${ }^{22}$ Preliminary measurements show that, after the transition, the $E \times B$ velocity shear is increased by a factor of 3 in the narrow region where the interchange mode is still observed but saturated at much lower amplitude. This increase leads to a ratio $\gamma /\left(\partial v_{E \times B} / \partial r\right)$ smaller than unity. Recent numerical simulations ${ }^{11}$ predict that a transition between a low confinement regime and a high confinement regime for $2 \mathrm{D}$ interchange-dominated simple magnetized plasmas occurs for a ratio close to 0.5 . Further comparisons with nonlinear simulations are under way.

\section{ACKNOWLEDGMENTS}

This work is partly funded by the Fonds National Suisse de la Recherche Scientifique. The authors acknowledge fruitful discussions with J. P. Graves.

${ }^{1}$ J. P. Freidberg, Ideal Magnetohydrodynamics (Plenum, New York, 1987). ${ }^{2}$ P. Ricci, B. N. Rogers, W. Dorland, and M. Barnes, Phys. Plasmas 13, 062102 (2006).

${ }^{3}$ J. Kesner, Phys. Plasmas 7, 3837 (2000).

${ }^{4}$ A. Simakov, P. J. Catto, and R. J. Hastie, Phys. Plasmas 8, 4414 (2001).

${ }^{5}$ A. Fasoli, B. Labit, M. McGrath, S. H. Müller, G. Plyushchev, M. Podestà, and F. M. Poli, Phys. Plasmas 13, 055902 (2006).

${ }^{6}$ F. M. Poli, S. Brunner, A. Diallo, A. Fasoli, I. Furno, B. Labit, S. H.
Müller, G. Plyushchev, and M. Podestà, Phys. Plasmas 13, 102104 (2006).

${ }^{7}$ F. M. Poli, P. Ricci, A. Fasoli, and M. Podestà, Phys. Plasmas 15, 032104 (2008).

${ }^{8}$ I. Furno, B. Labit, M. Podestà, A. Fasoli, S. H. Müller, F. M. Poli, P. Ricci, C. Theiler, S. Brunner, A. Diallo, and J. Graves, Phys. Rev. Lett. 100, 055004 (2008).

${ }^{9}$ C. Theiler, A. Diallo, A. Fasoli, I. Furno, B. Labit, M. Podestà, F. M. Poli, and P. Ricci, Phys. Plasmas 15, 042303 (2008).

${ }^{10}$ A. Zeiler, J. F. Drake, and B. Rogers, Phys. Plasmas 4, 2134 (1997).

${ }^{11}$ P. Ricci, B. Rogers, and S. Brunner, Phys. Rev. Lett. 100, 225002 (2008).

${ }^{12}$ A. E. Glassgold, P. S. Krstić, and D. R. Schultz, Astrophys. J. 621, 808 (2005).

${ }^{13} \mathrm{~L}$. Federspiel, "Investigation of the excitation threshold for pressure gradient driven instabilities in TORPEX plasmas", EPFL Master's Report, 2008 (unpublished).

${ }^{14}$ K. Rypdal and S. Ratynskaia, Phys. Plasmas 11, 10 (2004).

${ }^{15}$ M. Podestà, A. Fasoli, B. Labit, M. McGrath, S. H. Müller, and F. M. Poli, Plasma Phys. Controlled Fusion 47, 1989 (2005).

${ }^{16}$ S. H. Müller, A. Fasoli, B. Labit, M. McGrath, O. Pisaturo, G. Plyushchev, M. Podestà, and F. M. Poli, Phys. Plasmas 12, 090906 (2005).

${ }^{17}$ J. M. Beall, Y. C. Kim, and E. J. Powers, J. Appl. Phys. 53, 3933 (1982).

${ }^{18}$ N. Iwama and T. Tsukishima, Appl. Phys. Lett. 31, 783 (1977).

${ }^{19}$ F. M. Poli, M. Podestà, and A. Fasoli, Rev. Sci. Instrum. 80, 053501 (2009).

${ }^{20}$ F. M. Poli, Ph.D. thesis, EPFL, 2007.

${ }^{21}$ D. T. Garnier, A. C. Boxer, J. L. Ellsworth, A. K. Hansen, I. Karim, J. Kesner, M. E. Mauel, E. E. Ortiz, and A. Roach, J. Plasma Phys. 74, 733 (2008).

${ }^{22}$ P. W. Terry, Rev. Mod. Phys. 72, 109 (2000). 